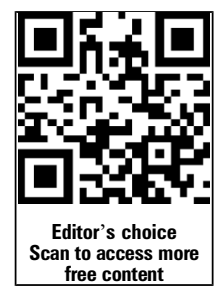

${ }^{1}$ Department of Emergency Medicine, The University of Texas Health Science Center Medical School, Houston,

Texas, USA

${ }^{2}$ Houston Veterans Affairs Center for Innovations in Quality, Effectiveness and Safety, Michael E. DeBakey Veterans Affairs Medical Center Houston, Texas, USA ${ }^{3}$ Section of Health Services Research, Department of Medicine, Baylor College of Medicine, Houston, TX, USA

\section{Correspondence to}

Dr Nnaemeka Okafor, Department of Emergency Medicine, The University of Texas Health Science Center Medical School, 5431 Fannin Street, Houston, TX 77030, USA:

Nnaemeka.G.Okafor@uth. tmc.edu

Received 22 December 2014 Revised 3 August 2015 Published Online First 4 November 2015

\title{
Using voluntary reports from physicians to learn from diagnostic errors in emergency medicine
}

\author{
Nnaemeka Okafor, ${ }^{1}$ Velma L Payne, ${ }^{2,3}$ Yashwant Chathampally, ${ }^{1}$ Sara Miller, ${ }^{1}$ \\ Pratik Doshi, ${ }^{1}$ Hardeep Singh ${ }^{2,3}$
} Accepted 25 August 2015

\begin{abstract}
Objectives Diagnostic errors are common in the emergency department (ED), but few studies have comprehensively evaluated their types and origins. We analysed incidents reported by ED physicians to determine disease conditions, contributory factors and patient harm associated with ED-related diagnostic errors.

Methods Between 1 March 2009 and 31 December 2013, ED physicians reported 509 incidents using a department-specific voluntary incident-reporting system that we implemented at two large academic hospitalaffiliated EDs. For this study, we analysed 209 incidents related to diagnosis. A quality assurance team led by an ED physician champion reviewed each incident and interviewed physicians when necessary to confirm the presence/absence of diagnostic error and to determine the contributory factors. We generated descriptive statistics quantifying disease conditions involved, contributory factors and patient harm from errors. Results Among the 209 incidents, we identified 214 diagnostic errors associated with 65 unique diseases/ conditions, including sepsis $(9.6 \%)$, acute coronary syndrome $(9.1 \%)$, fractures $(8.6 \%)$ and vascular injuries $(8.6 \%)$. Contributory factors included cognitive $(n=317)$,

\section{Key messages}

What is already known on this subject?

- Diagnostic errors are common in the emergency department (ED), but few studies have comprehensively evaluated their types and origins.

- Limited studies have examined the role of incident reporting systems in the study of diagnostic errors and none in the ED setting.

What might this study add?

- ED physicians voluntarily reported 509 incidents between 2009 and 2013, of which, 209 were related to diagnostic errors involving a variety of common diseases.

- Contributory factors identified included cognitive $(n=317)$, system related $(n=192)$ and non-remedial $(n=106) ; 75 \%$ of the incidents involved multiple factors.

- The programme led to diagnostic error prevention strategies, positioning this body of work as a useful foundation for other institutions.
\end{abstract} system related $(n=192)$ and non-remedial $(n=106)$. Cognitive factors included faulty information verification $(41.3 \%)$ and faulty information processing $(30.6 \%)$ whereas system factors included high workload (34.4\%) and inefficient ED processes (40.1\%). Non-remediable factors included atypical presentation (31.3\%) and the patients' inability to provide a history (31.3\%). Most errors $(75 \%)$ involved multiple factors. Major harm was associated with 34/209 (16.3\%) of reported incidents.

Conclusions Most diagnostic errors in ED appeared to relate to common disease conditions. While sustaining diagnostic error reporting programmes might be challenging, our analysis reveals the potential value of such systems in identifying targets for improving patient safety in the ED.

- http://dx.doi.org/10.1136/ emermed-2014-204564 - http://dx.doi.org/10.1136/ emermed-2015-204754 - http://dx.doi.org/10.1136/ emermed-2016-205696 CrossMark

To cite: Okafor $\mathrm{N}$ Payne VL, Chathampally $Y$ et al. Emerg Med $\mathrm{J}$ 2016:33:245-252.

\section{INTRODUCTION}

The practice of emergency medicine (EM) is considered 'a natural laboratory for the study of error'. ${ }^{2}$ Brief patient-physician encounters for patients who might be severely ill occur in the midst of high physician demands in a busy emergency department (ED) setting, thus creating an environment rich for error. ${ }^{1-5}$ According to the Institute of Medicine's To Err is Human report, $70 \%$ of ED-based errors are preventable. ${ }^{6}$ A 2006 Institute of Medicine report on hospital-based emergency care indicated that EDs are 'at the breaking point' due to being highly fragmented, overburdened and underfunded. ${ }^{7}$

Diagnostic error is likely to be one of the most common types of errors in the ED setting. ${ }^{3}{ }^{4} 8-10$ A study of closed malpractice claims found that $65 \%$ of the claims involved missed ED diagnoses, of which, 48\% caused serious harm, and 39\% resulted in death. ${ }^{10}$ Diagnostic errors have been difficult to detect and understand. ${ }^{11-13}$ One method that could promote learning about diagnostic errors is physician reporting, ${ }^{14}$ although most errors in clinical practice are never reported, and there is insufficient empiric literature on this topic. For example, there are limited studies examining the role of incident-reporting systems in the study of diagnostic errors, and none are in the ED setting. Furthermore, studies have shown that only $2 \%$ of incident reports are from physicians. ${ }^{15} 16$ Voluntary reporting offers the unique opportunity to explore both system-related and cognitive factors contributing to diagnostic errors, ${ }^{11} 1718$ especially because physicians are in a unique position to explain judgement lapses resulting in inaccurate diagnosis. In order for voluntary reporting systems to be used, they must be simple, minimise inconvenience, be non-punitive and maintain reporter confidentiality. ${ }^{16}{ }^{19}$ Incidents must be analysed by an unbiased 
expert panel, and timely feedback must be given to those involved for reporting to be impactful, but this does not always happen in clinical practice. ${ }^{162021}$

To overcome some of the challenges to physician reporting described above, we developed and implemented a voluntary incident-reporting system and created a robust error registry to facilitate continuous analysis of ED medical errors. The system was used to provide physicians with timely, direct and individualised feedback, including strategies to minimise recurrent errors. The voluntary non-punitive incident-reporting system was implemented after a campaign to encourage physicians to (1) acknowledge the ED as an error-prone environment; (2) report near misses, adverse events and medical errors and (3) increase collaboration with ED staff and other medical disciplines to establish a culture of safety. Access to the reporting system was limited to ED attendings, resident physicians and advanced practitioners. In this study, we analysed the data from these incident reports to determine the disease conditions, contributory factors and patient harm associated with ED-related diagnostic errors. Such studies could lead to creation and dissemination of strategies to minimise these errors.

\section{METHODS}

\section{Study settings}

A physician champion supported by the ED quality assurance (QA) committee developed and implemented a voluntary medical error reporting system at the two academic sites affiliated with the University of Texas Health Science Center Medical School (UTHealth). ${ }^{22}$ We aimed to collect and analyse ED medical errors to identify the latent contributing factors and suggest methods to decrease errors and harm. Site A was a tertiary referral, level 1 trauma centre with $35 \mathrm{ED}$ beds and an annual volume of $60000 \mathrm{ED}$ patient visits and on average a $40 \%$ admission rate during the study period, staffed primarily with EM faculty and residents. Site B was an urban county hospital with $45 \mathrm{ED}$ beds and an annual volume of $74000 \mathrm{ED}$ patient visits and on average a $16 \%$ admission rate during the study period, staffed by EM faculty, advanced practice professionals and EM residents (physicians in training). Within our two study settings, 30 attending (staff) physicians, 35 advanced practice professionals and 54 supervised resident physicians care for approximately $150000 \mathrm{ED}$ patient visits. Over the study period, the faculty and residents worked at both sites. Both hospitals have integrated, well-established electronic health record (EHR) systems and provide care to ethnically and socioeconomically diverse patients from rural and urban areas. Faculty physicians, advanced practice professionals and residents were encouraged to report any medical error that they felt merited review. For this study, we conducted a retrospective review of 209 unique diagnostic errors reported between 1 March 2009 and 31 December 2013. Reported errors that were not diagnosis related were not reviewed in this study. The study was approved by the local institutional review boards.

With the rapid growth of quality and safety initiatives nationwide, many hospitals, including our own, have become very supportive of patient safety improvement systems. Our primary residency hospital has created a Quality and Safety Academy for the purpose of annually training physicians and nurses from each department in improvement science and processes. The hospital has multiple ongoing safety programmes focusing on reducing catheter-related infections, ventilator-associated pneumonia, patient falls as well as implementation of sepsis identification tools and best practice pathways for trauma, stroke and ST elevation myocardial infarction care. The hospital requires the ED to complete monthly chart reviews based on the following event triggers: death in the ED, unscheduled return within $72 \mathrm{~h}$ and escalation of care of the admitted patient due to deterioration within $24 \mathrm{~h}$ of ED departure. The UTHealth ED staff initiated the same quality initiatives and safety culture at the second site.

Our QA process is protected from legal discovery. Nevertheless, the EM QA committee recognised that the fear of malpractice could contribute to under-reporting by certain providers and that it would be difficult to completely remove the fear of potential professional liability. ${ }^{23}$ Over the long term though, our non-punitive system led to increased reporting. ${ }^{22}$

\section{Incident review process}

A multidisciplinary QA committee of ED physicians, physician assistants and nurses, who were familiar with both ED sites, reviewed the reported incidents and provided feedback to the involved and reporting clinicians. Core UTHealth EM faculty members of the QA committee who worked at both sites received additional safety training. The QA committee promoted a safety culture using monthly lectures and feedback from reported cases. Briefly, the incident reporting and review process was used for both sites and consisted of five steps (figure 1).

Step 1: once an error was identified, physicians logged on to the system and entered a short narrative of the incident. Incidents were identified and reported in any of the following ways:

1. On a return visit to the ED, the patient is evaluated by the same physician who discovers an error and self-reports an incident.

2. A second care team involved in a return ED visit or a shift change discovers a possible error and reports the incident.

3. The inpatient care team postadmission identifies an error made by the ED and reports it to the ED medical director who would log the incident in the reporting system.

4. An error is discovered on a routine review conducted by our ED on all return visits to the ED within $72 \mathrm{~h}$ or death within $24 \mathrm{~h}$ of admission. These incidents are also reported to the ED medical director who enters them into the reporting system.

Errors involving residents were only classified as a diagnostic error if the entire care team, including the supervising attending physician, did not recognise the error. Most incidents were reported by physicians who discovered an error made by someone else, but some also by physicians who made the error. To obtain feedback from sources external to the ED, we encouraged the leaders and key personnel in other specialties to refer ED-related medical errors to us. However, the majority of errors were referred by ED physicians themselves. On submission, all incidents were stored in a secured database accessible only by the QA committee.

Step 2: once an incident was reported, the physician(s) named in the incident immediately received an email notifying them that an incident had been logged. They were asked to complete a Physician Incident Report Form (PIRF) detailing their account of the incident, acuity of the patient and the ED work environment at the time of the incident and to select the cognitive, system-related or non-remedial factors that they felt contributed to the incident. PIRFs were instrumental in providing key information regarding the incident. Real-time notification and the $72 \mathrm{~h}$ and subsequent reminders aimed to decrease physician recall bias. A member of the QA committee monitored pending 


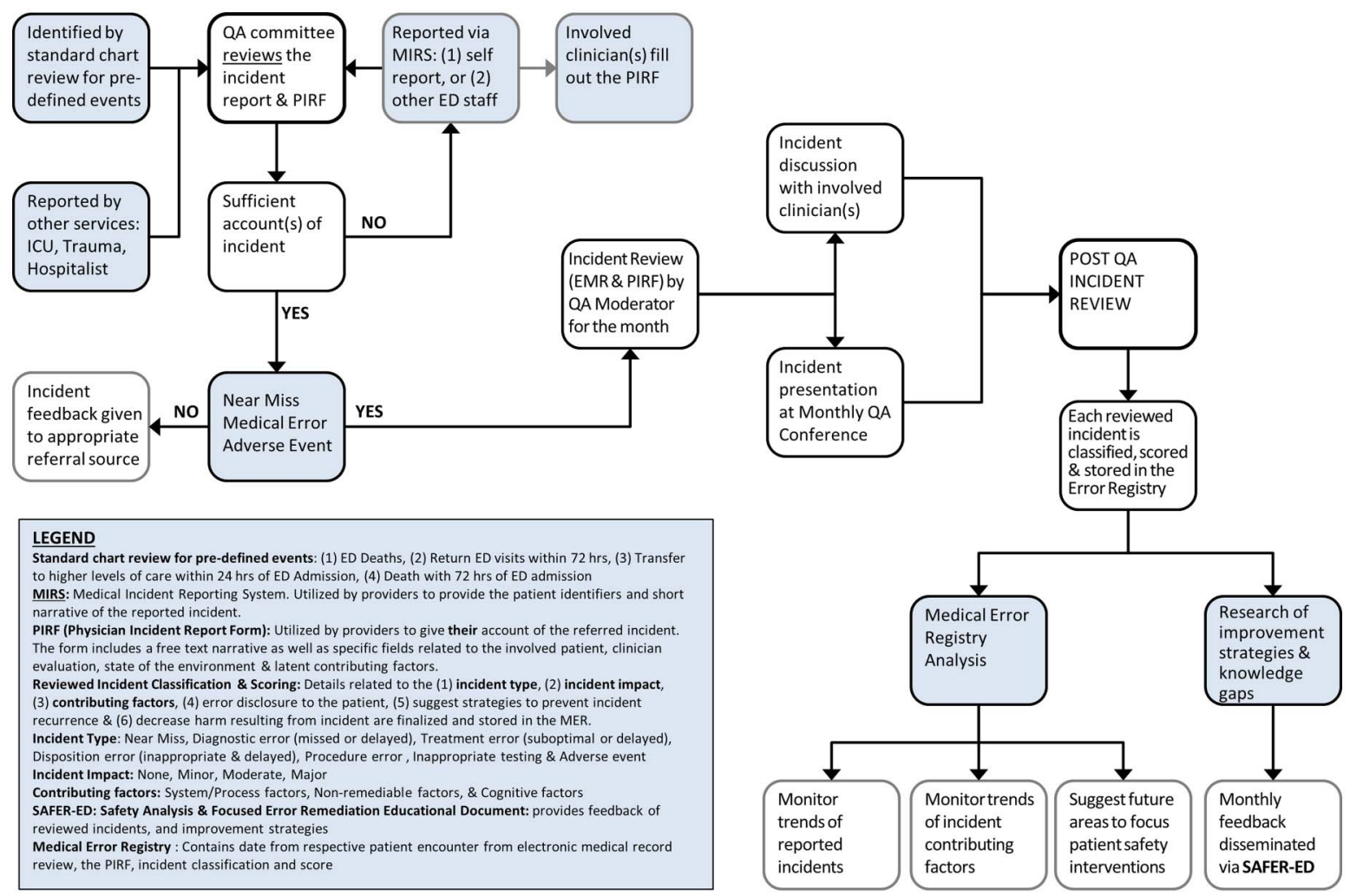

\section{Quality Assurance Process Map}

Figure 1 Flowchart of QA incident review. ED, emergency department; ICU, intensive care unit; QA, quality assurance.

incidents and encouraged physicians to complete the PIRF on a timely basis.

Step 3: once the PIRF was available, the QA committee reviewed the incident report, PIRF and the patient's medical record in detail. The committee consisted of four experienced attending physicians with deep understanding of the ED processes, one physician assistant and one nurse, but occasionally, resident physicians and additional members participated as 'ad hocs' to learn how to perform root cause analyses of reported incidents. During this initial review, the incident was classified according to the Institute of Medicine error categories as 'No Error', 'Near Miss' (defined as an unplanned event that did not result in injury, illness or damage, but had the potential to do so), 'Adverse Event' (defined as an injury caused by medical management rather than the underlying condition of the patient) or 'Medical Error' (defined as the failure of a planned action to be completed as intended or the use of a wrong plan to achieve an aim). ${ }^{6}$ The committee also discussed the contributory factor selected by the involved physician and modified it if necessary. Differences in opinion were resolved by committee discussion and consensus.

Step 4: for each confirmed error, a QA moderator (one of the attending physician members who previously reviewed the case) performed an in-depth review of the medical record and additional case details obtained previously to identify the contributing factors and the presence of patient harm. If the moderator needed clarifying information, such as additional details of the ED environment during the patient encounter or the patient's mental status or capacity, he/she contacted the physician for additional information. Once the review was complete, the QA committee reconvened to finalise the error classification, patient harm and contributing factors. The committee also compiled a list of strategies to potentially prevent incident recurrence and patient harm. Findings of the investigation were documented on a QA Incident Review Form. Confirmed error incidents were stored in a medical error registry database.

Errors were classified as procedure error, diagnostic error, inappropriate disposition, inappropriate or delayed therapy, inappropriate testing or other. An incident was classified as a diagnostic error only when it was clear from the QA committee review of the medical record and the involved clinician incident report that adequate information was available to make the diagnosis in the ED, but the information was not used, acted upon or interpreted correctly during the diagnostic process. For instance, if a patient with sepsis failed to receive timely and/or appropriate resuscitation (antibiotics, fluids, vasopressors), and it was determined that the involved clinicians did not consider the diagnosis of sepsis or pursue additional appropriate evaluations or actions related to sepsis despite having the data necessary to do so, we would label that as a diagnostic error.

Patient harm assigned at this stage was designated as minor (patient inconvenience, dissatisfaction or increased length of stay), moderate (short-term morbidity, disposition to a higher level of care, the need for an invasive procedure), major (death, permanent disability or near life-threatening or limb-threatening event), none or unknown. Patient harm categories were slightly modified for the ED setting using the taxonomy proposed by Schiff et $a .^{24}$ Patient harm was determined by evaluating the clinical impact of the diagnostic error on the patient while accounting for the underlying disease process, as well as expected mortality.

Contributory factors were adapted from Graber's taxonomy, categorised as cognitive, system related or non-remedial. ${ }^{25}$ Cognitive factors were subdivided as faulty knowledge, faulty 
data gathering, faulty information processing, faulty information verification. System-related factors included equipment failure, software failure, poor equipment usability, high workload, inefficient process (eg, turnaround time from test order to test result took longer than expected), insufficient resources, inadequate handoff, non-handoff communication error or other. We relied on multiple sources of data to make these determinations and obtained confirmatory evidence for these factors when possible, for example, handoff adequacy was determined by conversation with both parties involved. A handoff was considered inadequate when there was a communication error involving incorrect representation or omission of pertinent information such as vital signs, diagnostic orders, diagnostic order results available and pending and/or specification of a differential diagnosis. We determined factors as non-remedial when patients presented with atypical or rare conditions, or were unable to give an accurate history. This could occur due to factors such as dementia, psychiatric issues or an altered mental status with no family available for collateral history, language barriers and patient non-adherence. While it was possible for non-remedial factors to be addressed in the long run, we made this determination as applicable at the time of patient care. High workload was determined via physician self-report. Findings of the investigation were documented on the QA Incident Review Form. Confirmed error incidents were stored in a medical error registry database.

Step 5: in order to facilitate learning, reduce the potential of recurring incidents and mitigate the negative association of error reporting, illustrative de-identified cases were presented at a monthly QA meeting and published in a monthly QA newsletter.

\section{Data analysis}

We generated descriptive statistics to quantify the frequency of clinical conditions associated with diagnostic errors, contributory factors and patient harm.
RESULTS

Of the 509 reviewed incidents reported between 1 March 2009 and 31 December 2013, 209 (42\%) incidents were diagnosis related (figure 2). Some cases had more than one missed diagnosis, resulting in a total of 214 diagnostic errors. There were 65 unique diagnoses missed, with sepsis $(9.6 \%)$, acute coronary syndrome $(9.1 \%)$, fractures $(8.6 \%)$, vascular injuries $(8.6 \%)$, cerebrovascular accident (3.8\%), cardiac dysrhythmia (2.9\%), non-septic shock $(2.9 \%)$, hypoglycaemia $(2.9 \%)$, ectopic pregnancy $(2.4 \%)$, electrolyte derangement $(2.4 \%)$, pericardial effusion $(2.4 \%)$, pneumonia $(2.4 \%)$ and pulmonary embolism $(2.4 \%)$ being the most common (table 1$)$. While error in diagnosis of any of the conditions listed in table 1 has the potential to cause adverse events, not all of the diagnostic error incidents we analysed resulted in actual patient harm.

Cognitive factors were a major contributor to diagnostic errors occurring in the ED. In the 209 incidents, we identified 317 cognitive factors (table 2). Faulty information verification was a contributing factor in $130(41 \%)$ cognitive errors, faulty information processing occurred in 97 (30.7\%), faulty data gathering in $61(19.2 \%)$ and faulty knowledge contributed to $29(9.1 \%)$ cognitive errors. Table 2 lists examples of each type.

System-related factors impacted the patient visit in 192 incidents (table 3). Inefficient processes, including diagnostic test processing delays or delays in critical results notification by radiology, ranked highest, occurring in 77 (40.0\%) incidents. High workload in the ED was a factor in 66 (34.4\%) incidents. Inadequate handover occurred in 19 (9.9\%) cases. Insufficient resources contributed to 17 (8.9\%) errors and involved bed availability (eg, inadequate assessment in hallway when ED beds were unavailable), equipment such as ECG machines not readily available or staffing shortages, all of which resulted in the inability to attend to incoming ED patients. Poor equipment usability (a clinical information system with poor interface that did not convey to providers that important additional information was available by scrolling down) contributed to $4(2.1 \%)$ errors.

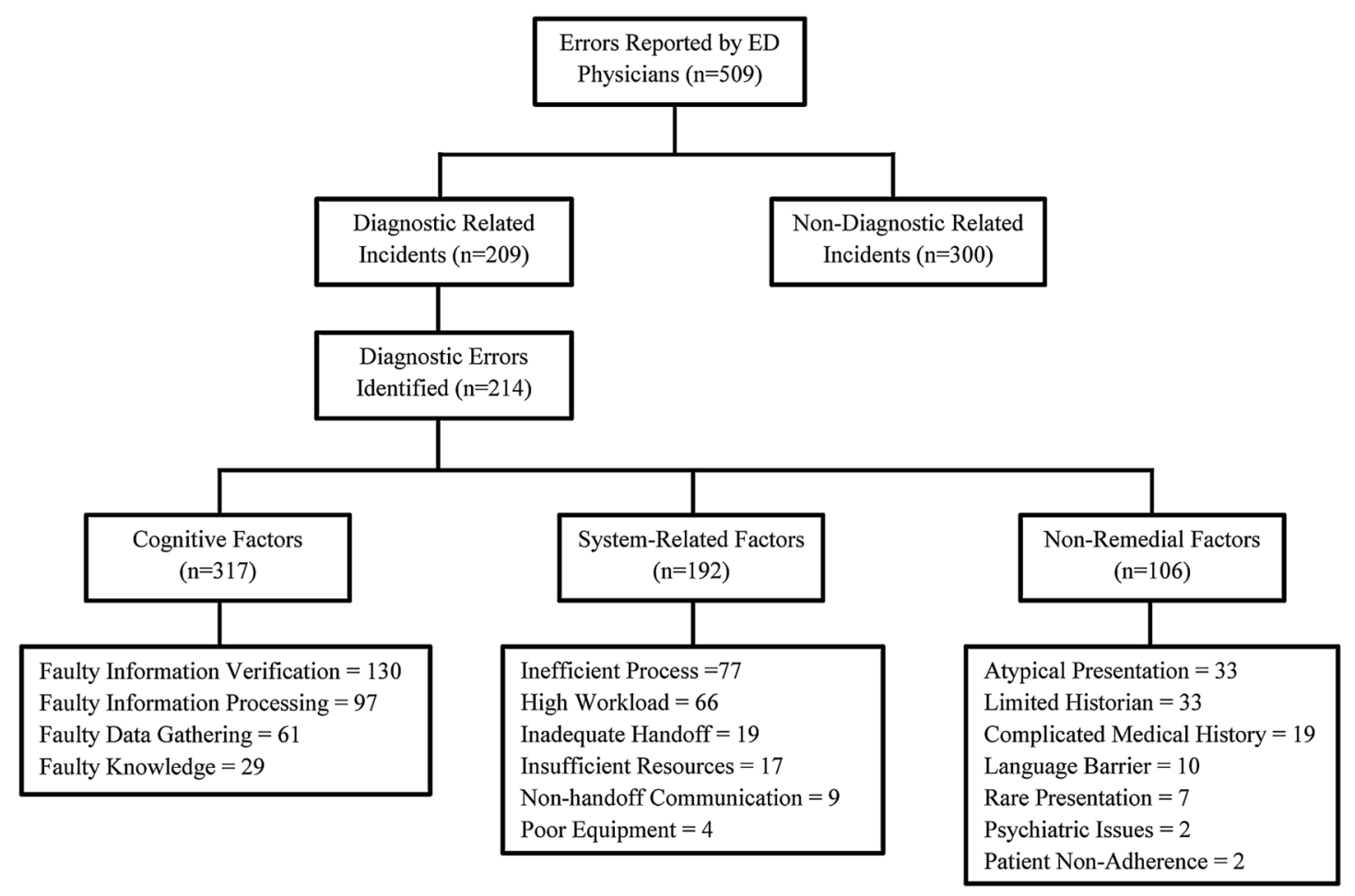

Figure 2 Incident flow diagram. ED, emergency department. 
Table 1 Diagnostic error frequency

\begin{tabular}{|c|c|}
\hline Disease & $\begin{array}{l}\text { Number of cases }(\%) \\
(\mathrm{N}=209)\end{array}$ \\
\hline Sepsis & $20(9.6)$ \\
\hline Acute coronary syndrome & $19(9.1)$ \\
\hline Fractures-vertebral spine, extremity, rib or pelvic & $18(8.6)$ \\
\hline Vascular injury* & $18(8.6)$ \\
\hline Cerebrovascular accident & $8(3.8)$ \\
\hline Cardiac dysrhythmia & $6(2.9)$ \\
\hline Non-septic shock & $6(2.9)$ \\
\hline Hypoglycaemia & $6(2.9)$ \\
\hline Ectopic pregnancy & $5(2.4)$ \\
\hline Electrolyte derangement & $5(2.4)$ \\
\hline Pericardial effusion & $5(2.4)$ \\
\hline Pneumonia & $5(2.4)$ \\
\hline Pulmonary embolism & $5(2.4)$ \\
\hline Abscess & $4(1.9)$ \\
\hline Appendicitis & $4(1.9)$ \\
\hline Bowel injury & $4(1.9)$ \\
\hline Gastrointestinal bleeding & $4(1.9)$ \\
\hline Coagulopathy & $3(1.4)$ \\
\hline Haemoperitoneum & $3(1.4)$ \\
\hline Intestinal malrotation & $3(1.4)$ \\
\hline Meningitis & $3(1.4)$ \\
\hline Peritonitis & $3(1.4)$ \\
\hline Rh-negative status & $3(1.4)$ \\
\hline Small bowel obstruction & $3(1.4)$ \\
\hline Cerebral oedema & $2(1.0)$ \\
\hline Cholecystitis & $2(1.0)$ \\
\hline Diabetic ketoacidosis & $2(1.0)$ \\
\hline Epidural haematoma & $2(1.0)$ \\
\hline Hypoxia & $2(1.0)$ \\
\hline Intraocular foreign body & $2(1.0)$ \\
\hline Subarachnoid haemorrhage & $2(1.0)$ \\
\hline Subdural haematoma & $2(1.0)$ \\
\hline Testicular injury & $2(1.0)$ \\
\hline Urinary tract infection/pyelonephritis & $2(1.0)$ \\
\hline Acute closure glaucoma & $1(0.5)$ \\
\hline Angioedema & $1(0.5)$ \\
\hline Autonomic dysreflexia & $1(0.5)$ \\
\hline Brugada syndrome & $1(0.5)$ \\
\hline Cancer & $1(0.5)$ \\
\hline Cardiac injury & $1(0.5)$ \\
\hline Central vertigo & $1(0.5)$ \\
\hline Complex migraine & $1(0.5)$ \\
\hline Cranial nerve palsy & $1(0.5)$ \\
\hline Hirschsprung enterocolitis & $1(0.5)$ \\
\hline Hyperglycaemia & $1(0.5)$ \\
\hline Hypokalemic periodic paralysis & $1(0.5)$ \\
\hline Infected kidney stone & $1(0.5)$ \\
\hline Intra-abdominal bleeding & $1(0.5)$ \\
\hline Intracranial shunt malfunction & $1(0.5)$ \\
\hline Laryngeal mass & $1(0.5)$ \\
\hline Nephrotic syndrome & $1(0.5)$ \\
\hline Demyelinating disease & $1(0.5)$ \\
\hline Neutropenia & $1(0.5)$ \\
\hline Ovarian torsion & $1(0.5)$ \\
\hline Pericarditis & $1(0.5)$ \\
\hline Pleural effusion & $1(0.5)$ \\
\hline Pulmonary contusion & $1(0.5)$ \\
\hline Pregnancy & $1(0.5)$ \\
\hline
\end{tabular}

Table 1 Continued

\begin{tabular}{lc}
\hline Disease & $\begin{array}{l}\text { Number of cases (\%) } \\
(\mathrm{N}=\mathbf{2 0 9 )}\end{array}$ \\
\hline Pulmonary oedema & $1(0.5)$ \\
Spinal cord compression & $1(0.5)$ \\
Strangulated abdominal hernia & $1(0.5)$ \\
Symptomatic anaemia & $1(0.5)$ \\
Urethral injury & $1(0.5)$ \\
Urinary retention & $1(0.5)$ \\
Worsening brain metastasis & $1(0.5)$ \\
\hline *Aortic dissection, aortic arch injury, carotid dissection, extremity arterial \\
insufficiency, inferior epigastric arterial injury, ruptured abdominal aortic aneurysm, \\
superior mesenteric artery injury, vertebral artery dissection.
\end{tabular}

Non-remedial errors occurred in 106 incidents (table 4) and included atypical presentation (31.3\%) and the patients' inability to provide a history $(31.3 \%)$.

Three-quarters of incidents $(\mathrm{n}=157,75 \%)$ involved multiple types of contributory factors (figure 3). The Venn diagram depicts the distribution of cognitive, system-related and nonremedial factors across the 209 incidents. Each incident was reviewed to determine if it contained a cognitive, system-related and/or non-remedial factor and assigned to a single category. All three factors were present in $53(25 \%)$ cases.

The potential severity of harm was major (ie, resulting in a life-threatening or limb-threatening event, permanent disability or death) for $34(16.3 \%)$ patients. Examples of major harm included patients who died as a result of sepsis due to its delayed recognition and thus treatment. Moderate harm, defined as short-term morbidity, disposition to a higher level of care or the need for an invasive procedure, occurred in 66 $(31.6 \%)$ cases. For example, patients were admitted to the intensive care unit instead of an intermediate care unit due to delayed resuscitation that resulted from diagnostic errors.

Table 2 Cognitive factors contributing to diagnostic errors

\begin{tabular}{|c|c|c|}
\hline $\begin{array}{l}\text { Contributory } \\
\text { factor }\end{array}$ & $\begin{array}{l}\text { Number of } \\
\text { errors }(\%) \\
(\mathrm{N}=317)\end{array}$ & Example \\
\hline $\begin{array}{l}\text { Faulty information } \\
\text { verification }\end{array}$ & $130(41.0)$ & $\begin{array}{l}\text { Premature closure occurred in a } \\
\text { patient who presented with } \\
\text { generalised weakness. He was thought } \\
\text { to have the diagnosis of coronary } \\
\text { ischaemia and laboratory testing to } \\
\text { check for hypopotassaemia was not } \\
\text { ordered until much later }\end{array}$ \\
\hline $\begin{array}{l}\text { Faulty information } \\
\text { processing }\end{array}$ & $97(30.7)$ & $\begin{array}{l}\text { There was misinterpretation of } \\
\text { diagnostic clinical data in a patient } \\
\text { with known renal disease who } \\
\text { presented with hypotension. The care } \\
\text { team initially suspected overdiuresis, } \\
\text { but the patient actually had sepsis }\end{array}$ \\
\hline $\begin{array}{l}\text { Faulty data } \\
\text { gathering }\end{array}$ & $61(19.2)$ & $\begin{array}{l}\text { Patient presented with a lesion on the } \\
\text { back, which was missed by the initial } \\
\text { care team. He was later discovered to } \\
\text { have an extensive infection }\end{array}$ \\
\hline Faulty knowledge & $29(9.1)$ & $\begin{array}{l}\text { A patient presented with hip pain and } \\
\text { was found to have negative hip X-rays. } \\
\text { He was later discovered to have distal } \\
\text { femur fracture (which sometimes can } \\
\text { cause referred hip pain) on knee } \\
\text { X-rays. }\end{array}$ \\
\hline
\end{tabular}


Table 3 System-related factors contributing to diagnostic errors

\begin{tabular}{ll} 
Contributory factor & Number of errors (\%) (N=192) \\
\hline Inefficient process & $77(40.0)$ \\
High workload & $66(34.4)$ \\
Inadequate handoff & $19(9.9)$ \\
Insufficient resources & $17(8.9)$ \\
Non-handoff communication error & $9(4.7)$ \\
Poor equipment usability & $4(2.1)$ \\
\hline
\end{tabular}

Seventy-two (34.4\%) patients experienced minor harm, defined as an increased length of stay in the ED or hospital, patient inconvenience or dissatisfaction. For example, patients experienced prolonged ED and hospital stays as a result of an inappropriate or delayed recognition of the correct diagnosis. No harm was found in $19(9.1 \%)$ patients. We were unable to determine the presence or absence of harm or its relationship with diagnostic error in $18(8.6 \%)$ patients.

\section{DISCUSSION}

To advance the body of knowledge on types and origins of ED-related diagnostic errors, we analysed 209 diagnostic errorrelated incidents voluntarily reported by ED physicians. Diagnostic errors were associated with a variety of conditions commonly seen in the ED. About one-sixth of patients experienced major harm and an additional third experienced moderate harm. Nearly half of the incidents involved both cognitive and system-related factors, and one-fourth involved all three factor categories: cognitive, system related and non-remedial.

Our study builds on previous knowledge created from research on safety incident reports. The use of mandatory and voluntary incident reporting systems was strongly endorsed in the 1999 Institute of Medicine 'To Err is Human: Building a Safer Health System' report as an important component to improving patient safety. ${ }^{6}{ }^{16}$ Several studies have assessed voluntary report data, ${ }^{4} 14-16182026$ three of which reported on diagnostic errors. ${ }^{4} 1420$ A study from the UK assessed incident data reported by staff via a national reporting system collected over 2 years. ${ }^{14}$ They reported diagnostic error data specific to incident location, including the ED, but did not assess factors contributing to ED diagnostic errors. An error reporting system implemented at the Maine Medical Center was recently piloted within an adult inpatient medical service. ${ }^{20} 26$ The intent of the system was to capture diagnostic errors; however, diagnostic errors specific to the ED were not reported. The third study reviewed paper-based incidents reported by nurses, resident physicians, medical consultants and clerks working at 10 hospitals in the Netherlands. ${ }^{4}$ The authors reported on ED diagnostic

Table 4 Non-remedial factors contributing to diagnostic errors

\begin{tabular}{ll}
\hline Contributory factor & Number of errors (\%) (N=106) \\
\hline Atypical presentation & $33(31.1)$ \\
Limited historian & $33(31.1)$ \\
Complicated medical history & $19(18.0)$ \\
Language barrier & $10(9.4)$ \\
Rare presentation & $7(6.6)$ \\
Psychiatric issues & $2(1.9)$ \\
Patient non-adherence & $2(1.9)$ \\
\hline
\end{tabular}

errors, but statistics for diagnostic and treatment errors were combined. While other studies have assessed ED errors through malpractice claims, ${ }^{10}$ ED staff interviews, ${ }^{9}$ downstream reports by hospital ward physicians ${ }^{3}$ and mortality and morbidity reports, ${ }^{8}$ none of these studies focused on diagnostic errors. Our study is unique in that we specifically focused our analysis on diagnostic-related incidents voluntarily reported by ED physicians that occurred in the ED.

While few studies have comprehensively assessed reported data related to diagnostic errors, none have evaluated contributory factors to promote learning. To our knowledge, most EDs are not systematically analysing ED-specific diagnostic error incidents. Some EDs use hospital-wide reporting systems that are increasingly adopting the Agency for Healthcare Research and Quality common format, ${ }^{27}$ which does not have detailed categories for diagnostic error-related reporting. Additionally, our study strengths include the rich perspective of frontline personnel captured through voluntary reports from ED physicians as well as multidisciplinary team analysis. Our study also adds to the body of knowledge created by diagnostic error-related malpractice claims. ${ }^{28-31}$

A large variety of common types of diagnoses were missed, which is consistent with the malpractice literature. ${ }^{28-31}$ While types of diagnosis were largely similar to claims data (infections, fractures, acute coronary syndrome), a few new categories, such as vascular injuries and hypoglycaemia, emerged as common conditions. $^{26} \quad 30 \quad 32 \quad 33$ About half of these errors caused moderate-to-major harm, similar to primary care-related diagnostic errors. ${ }^{32}$ In the primary care setting, similar disease heterogeneity was found with a wide variety of common conditions that were missed.

Our findings illustrate the complexity of diagnostic incidents, where three-quarters had more than one contributory factor involved. It illustrates the challenges of information processing and information verification within a highly dynamic and busy ED environment. ${ }^{5}$ While cognitive interventions such as checklists and slowing-down strategies could play a role in reducing errors, process improvements in the ED setting would also be needed. ${ }^{34}$ For instance, notification of critical laboratory results was a contributing factor in $40 \%$ of the system-related errors, and process changes are often needed to make this more reliable. ${ }^{35}$ Similarly, managing other factors such as high workload and communication errors could reduce the vulnerability. Almost half of the incidents involved both cognitive and systemrelated factors, and it is likely that these factors interact in a complex fashion, and the effect of these factors is synergistic. Thus, multifaceted interventions are likely necessary to reduce ED diagnostic errors.

More than 200 diagnostic errors were reported, which suggests that ED physicians are aware that errors occur, and are willing to reduce them by reporting. However, this type of project requires physician champions to spend considerable time performing root cause analyses to determine the contributory factors and prevention strategies. Our project was valuable in making diagnostic errors a focus of quality improvement strategies in the ED (box 1). During the QA review process, recurrent diagnostic errors were identified and quality improvement strategies targeting at-risk processes were put into place. For example, the triage order and sign-out processes were revised and standardised. Treatment protocols were established for hypertension, head trauma, ectopic pregnancy and sickle cell disease. We established a common SharePoint 'one-stop-shop' site where clinicians could access departmental policies, clinical algorithms, protocols and guidelines. EHR tips-and-tricks 
Figure 3 Contributory factor relationship.

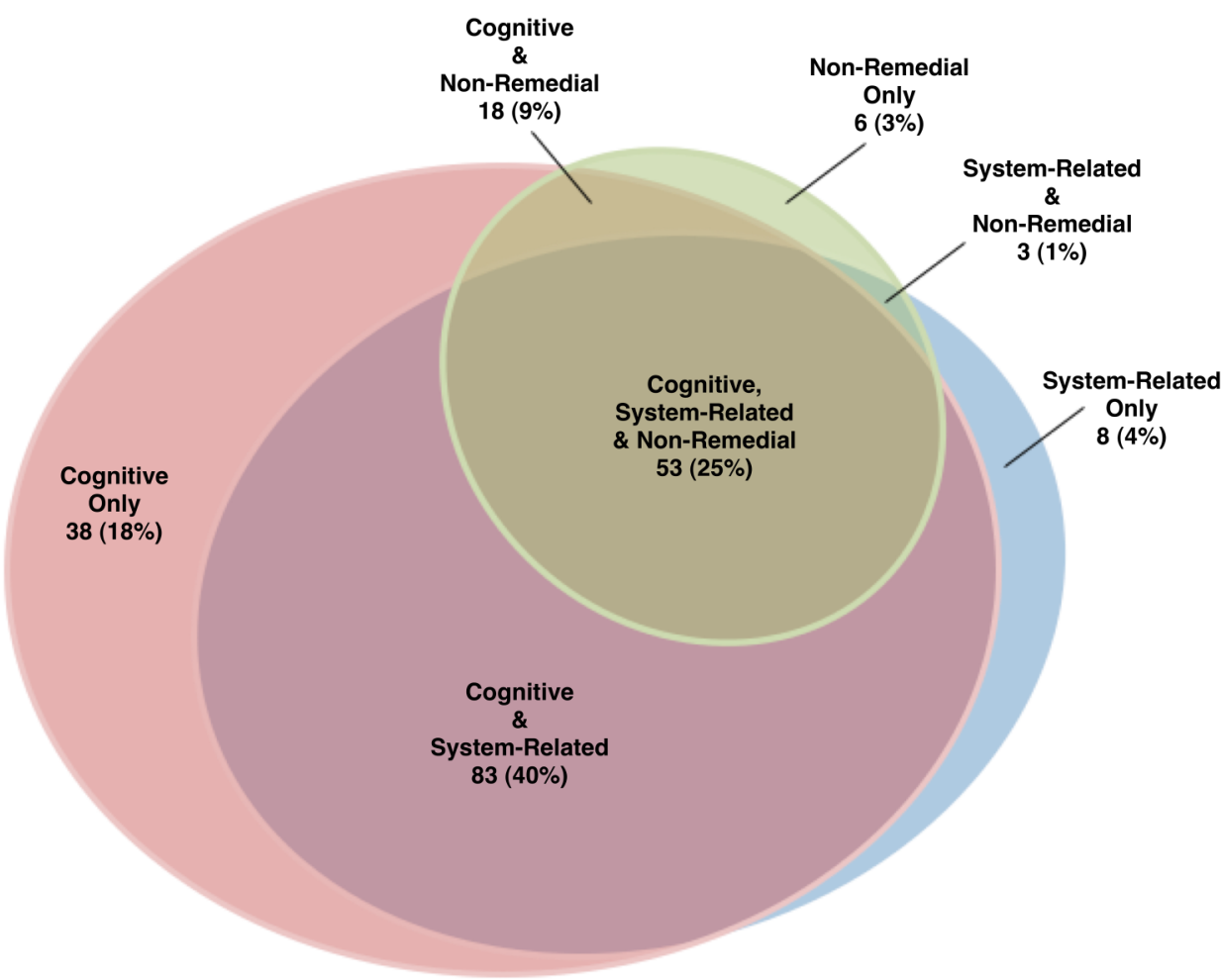

pamphlets were created and stored on this SharePoint site. Specific areas of vulnerabilities, such as ventilator management, reading ECGs and ultrasounds, were addressed through skill-and-knowledge-focused campaigns. The monthly mortality and morbidity conference was revamped, and a quarterly newsletter was developed to open communication and improve safety culture. Messages acknowledging the ED as an errorprone environment and promoting reporting of errors were strengthened.

\section{Box 1 Examples of error prevention strategies}

implemented as a result of the project

- Strategy description

- Standardisation of orders form to expedite care

- Standardisation of the sign-out process to include an independent review of all diagnostic orders and results during the sign-out process

- Establishment of treatment protocols for diseases such as hypertension, head trauma, ectopic pregnancy and sickle cell disease

- Establishment of a common SharePoint 'one-stop-shop' site where clinicians can access departmental policies, clinical algorithms, protocols and guidelines

- Creation of electronic health record tips-and-tricks pamphlets

- Skill-and-knowledge-focused campaigns conducted for specific areas of vulnerabilities, including ventilator management, ECG reading and ultrasounds

- Development of a quarterly newsletter to foster open communication of errors and to improve the culture of safety

- Incorporated discussions regarding acknowledging the emergency department as an error-prone environment and encouraging reporting errors into monthly morbidity and mortality conferences.

\section{Limitations}

Our work has several limitations. First, many diagnostic incidents were likely never reported. Physicians at disposition sites (eg, hospital ward physicians who cared for patients after admission from the ED) were not included; thus, we may have had fewer opportunities for identifying ED-based errors. Since the incidents were reported by physicians who are directly involved in patient care, it is possible that a bias towards reporting less significant errors with minimal patient impact may have resulted in patient harm being under-reported. However, almost onefifth of errors were still found to lead to severe harm, which is comparable with studies that did not involve reporting. ${ }^{3} 8$ Recall bias could also have impacted the level of detail reported if the reporter did not report the incident immediately after the event. We attempted to minimise recall bias by sending all personnel identified in the incident an automated system-generated email immediately after the incident was logged, requesting them to report their account of the incident. Similar to most studies of diagnostic error, our assessment might have suffered from hindsight bias ${ }^{11} 2425$ and some of our findings involved subjective judgements rather than objective measures. However, every effort was made to confirm assessments from multiple sources. For example, delays in test processing and physician workload were based on information provided in the incident reports and follow-up conversations with the physicians involved. A multilevel review process and use of a multidisciplinary team with a detailed chart review added strength to the reliability and validity of these judgements. Nevertheless, use of a QA committee with even a broader skill set (such as human factors experts and cognitive psychologists) might have resulted in a better characterisation of contributory factors. Finally, the distinction of contributory factors into categories of cognitive, system and non-remedial conveys an artificial distinction because physicians are making decisions in a dynamic and chaotic ED environment with a complex non-linear workflow. More prospective methods are needed to study the impact of environmental and systemic factors on physicians' cognition in 
the fast-paced, multitasking and interruption-laden ED environment. These methods could better explain judgement lapses resulting in inaccurate diagnoses. ${ }^{36}$ Lastly, in addition to leadership and institutional support, several clinical champions are required to jumpstart and sustain programmes to measure and improve diagnostic errors. ${ }^{37}$

\section{CONCLUSION}

In conclusion, voluntary ED error reports were useful to illustrate the types and origins of diagnostic errors. Our data suggest that physicians were willing to voluntarily report diagnostic errors. Several physician champions also invested a significant amount of time conducting root cause analyses to determine factors contributing to errors, which led to the development and implementation of intervention strategies. While sustaining diagnostic error reporting programmes might be challenging, our analysis reveals their potential value in identifying several areas ripe for improving patient safety in the ED. Given the distribution of cognitive and system contributory factors, multifaceted strategies are needed for the greatest impact in reducing diagnostic errors. We believe other institutions could develop and implement similar programmes to improve patient safety related to diagnostic errors.

\section{Twitter Follow Hardeep Singh at @ HardeepSinghMD}

Acknowledgements We would like to thank the clinicians who reported errors as well as those who served on the QA committee from 2009 through 2013 for their valuable contributions.

Contributors Study concept and design: NO, HS and VLP. Acquisition of data: NO, YC, SM and PD. Statistical analysis: NO, YC, SM and PD. Analysis and interpretation of data: NO, YC, SM, PD, VLP and HS. Drafting of the manuscript: VLP, HS, and NO. Critical revision of the manuscript for important intellectual content: NO, VLP YC, SM, PD and HS. Administrative, technical or material support: NO, VLP, YC, SM, PD and HS. Study supervision: VLP, HS and NO.

Funding This work was partially supported by a training fellowship from the Keck Center AHRQ Training Program in Patient Safety and Quality of the Gulf Coast Consortia (AHRQ Grant No. 1 T32HS017586-01). NO was supported by the AHRQ Training Program grant. VLP is supported by the VA Office of Academic Affiliations, Washington, DC and the Houston VA Center for Innovations in Quality, Effectiveness and Safety (CIN 13-413). HS is supported by the VA Health Services Research and Development Service (CRE 12-033; Presidential Early Career Award for Scientists and Engineers USA 14-274), the VA National Center for Patient Safety and the Agency for Health Care Research and Quality (R01HS022087) and partially supported by the Houston VA HSR\&D Center for Innovations in Quality, Effectiveness and Safety (CIN 13-413)

Disclaimer The views expressed in this article are those of the authors, and do not necessarily reflect the position or policy of any of the affiliated institutions, funders or the Department of Veterans Affairs.

Competing interests None declared.

Ethics approval The University of Texas IRB, Baylor IRB.

Provenance and peer review Not commissioned; externally peer reviewed.

\section{REFERENCES}

1 Croskerry P, Sinclair D. Emergency medicine: a practice prone to error? CJEM 2001;3:271-6.

2 Croskerry P, Shapiro M, Campbell S, et al. Profiles in patient safety: medication errors in the emergency department. Acad Emerg Med 2004;11:289-99.

3 Freund Y, Goulet H, Bokobza J, et al. Factors associated with adverse events resulting from medical errors in the emergency department: two work better than one. J Emerg Med 2013;45:157-62.

4 Smits M, Groenewegen PP, Timmermans DR, et al. The nature and causes of unintended events reported at ten emergency departments. BMC Emerg Med 2009;9:16.

5 Upadhyay DK, Sittig DF, Singh H. Ebola US Patient Zero: lessons on misdiagnosis and effective use of electronic health record. Diagnosis 2014;1:283-88.
6 Institute of Medicine. To err is human: building a safer health system. Washington, DC: National Academies Press, 1999.

7 Institute of Medicine. Hospital-based emergency care: at the breaking point. Washington, DC: National Academies Press, 2006

8 Cosby KS, Roberts R, Palivos L, et al. Characteristics of patient care management problems identified in emergency department morbidity and mortality investigations during 15 years. Ann Emerg Med 2008;51:251-61.

9 Fordyce J, Blank FS, Pekow P, et al. Errors in a busy emergency department. Ann Emerg Med 2003:42:324-33.

10 Kachalia A, Gandhi TK, Puopolo AL, et al. Missed and delayed diagnoses in the emergency department: a study of closed malpractice claims from 4 liability insurers. Ann Emerg Med 2007:49:196-205.

11 Graber ML. The incidence of diagnostic error in medicine. BMJ Qual Saf 2013;22 (Suppl 2):ii21-7.

12 Singh $\mathrm{H}$. Editorial: helping health care organizations to define diagnostic errors as missed opportunities in diagnosis. Jt Comm J Qual Patient Saf 2014;40:99-101.

13 Zwaan $\mathrm{L}$, Singh $\mathrm{H}$. The challenges in defining and measuring diagnostic error. Diagnosis 2015;2:97-103.

14 Sevdalis N, Jacklin R, Arora $\mathrm{S}$, et al. Diagnostic error in a national incident reporting system in the UK. J Eval Clin Pract 2010;16:1276-81.

15 Milch CE, Salem DN, Pauker SG, et al. Voluntary electronic reporting of medical errors and adverse events. An analysis of 92,547 reports from 26 acute care hospitals. J Gen Intern Med 2006;21:165-70.

16 Tuttle $D$, Holloway R, Baird T, et al. Electronic reporting to improve patient safety. Qual Saf Health Care 2004;13:281-6.

17 Graber ML. Taking steps towards a safer future: measures to promote timely and accurate medical diagnosis. Am J Med 2008;121:S43-6.

18 Kostopoulou O, Delaney B. Confidential reporting of patient safety events in primary care: results from a multilevel classification of cognitive and system factors. Qual Saf Health Care 2007;16:95-100.

19 Leape LL. Reporting of adverse events. N Engl J Med 2002;347:1633-8.

20 Trowbridge R, Salvador D. Addressing diagnostic errors: an institutional approach. National Patient Safety Foundation, 2010.

21 Karsh BT, Escoto KH, Beasley JW, et al. Toward a theoretical approach to medical error reporting system research and design. Appl Ergon 2006;37:283-95.

22 Okafor N, Chathampally Y, Miller S, et al. Use of a Voluntary Medical Incident Reporting tool as a Critical Component to Improve Physician Reporting of Medical Errors in an Emergency Department. Western Journal of Emergency Medicine 2015; in press.

23 Kaldjian LC, Jones EW, Rosenthal GE, et al. An empirically derived taxonomy of factors affecting physicians' willingness to disclose medical errors. I Gen Intern Med 2006;9:942-8.

24 Schiff GD, Hasan O, Kim S, et al. Diagnostic error in medicine: analysis of 583 physician-reported errors. Arch Intern Med 2009;169:1881-7.

25 Graber ML, Franklin N, Gordon R. Diagnostic error in internal medicine. Arch Intern Med 2005;165:1493-9.

26 Graber ML, Trowbridge R, Myers JS, et al. The next organizational challenge: finding and addressing diagnostic error. Jt Comm I Qual Patient Saf 2014;40:102-10.

27 AHRQ. AHRQ Common Format Website. https://www.pso.ahrq.gov/common

28 Brown TW, McCarthy ML, Kelen GD, et al. An epidemiologic study of closed emergency department malpractice claims in a national database of physician malpractice insurers. Acad Emerg Med 2010;17:553-60.

29 Holohan TV, Colestro J, Grippi J, et al. Analysis of diagnostic error in paid malpractice claims with substandard care in a large healthcare system. South Med J 2005;98:1083-7.

30 Phillips RL, Jr, Bartholomew LA, Dovey SM, et al. Learning from malpractice claims about negligent, adverse events in primary care in the United States. Qual Saf Health Care 2004;13:121-6.

31 Saber Tehrani AS, Lee H, Mathews SC, et al. 25-Year summary of US malpractice claims for diagnostic errors 1986-2010: an analysis from the National Practitioner Data Bank. BMJ Qual Saf 2013;22:672-80.

32 Singh $\mathrm{H}$, Giardina TD, Meyer AN, et al. Types and origins of diagnostic errors in primary care settings. JAMA Intern Med 2013;173:418-25.

33 Sonderegger-Iseli K, Burger S, Muntwyler J, et al. Diagnostic errors in three medical eras: a necropsy study. Lancet 2000;355:2027-31.

34 Graber ML, Sorensen AV, Biswas J, et al. Developing checklists to prevent diagnostic error in emergency settings. Diagnosis 2014;1:223-31.

35 Singh $\mathrm{H}, \mathrm{Vij}$ MS. Eight recommendations for policies for communicating abnormal test results. Jt Comm J Qual Patient Saf 2010;36:226-32.

36 Zwaan L, Schiff GD, Singh H. Advancing the research agenda for diagnostic error reduction. BMJ Qual Saf 2013;22(Suppl 2):ii52-7.

37 Singh H, Sittig DF. Advancing the science of measurement of diagnostic errors in healthcare: the safer Dx framework. BMJ Qual Saf 2015;24:103-10. 